\title{
EDIFÍCIO ESCOLAR VOLTADO ÀS INTELIGÊNCIAS MÚLTIPLAS E ÀS METODOLOGIAS ATIVAS
}

\author{
SQUAIELLA, Roberta B. F. \\ Universidade Presbiteriana Mackenzie, e-mail: robssquaiella@gmail.com \\ RIGHI, Roberto \\ Universidade Presbiteriana Mackenzie, e-mail: roberto.righi@mackenzie.br
}

\begin{abstract}
RESUMO
O presente artigo apresenta a discussão sobre a escola no século XXI, diante do impacto das tecnologias da informação e da comunicação. A pesquisa decorre do desenvolvimento de tese de doutorado em arquitetura e urbanismo, qualificada no final do ano de 2018 . O objetivo é identificar as novas configurações do edifício escolar, que darão o suporte para 0 desenvolvimento das Inteligências Múltiplas (IM), por meio das metodologias ativas. Na metodologia de pesquisa foi adotada a pesquisa de referências digitais e físicas, além do estudo de casos para caracterizar as IM e as metodologias ativas. Assim, foram identificadas as diferentes necessidades no uso do ambiente escolar e avaliados os projetos de edifícios escolares, que mostram o novo cenário do espaço educativo, em publicações de revistas especializadas e visitas em escolas inovadoras. Conclui-se, que uma nova configuração do ambiente escolar, com uma maior variedade de ambientes de aprendizagem potencializa $\circ$ desenvolvimento das IM. Tais ambientes devem favorecer as práticas pedagógicas mais colaborativas, em pequenas comunidades de aprendizagem, a partir de metodologias ativas. Para isso, o edifício escolar deve ser considerado como um todo, desde o seu acesso, até os ambientes para atividades mais especializadas. Espaços de circulação e local para refeições também devem ser incorporados como locais de aprendizagem. Novas diretrizes projetuais de edifícios escolares são necessárias para que arquitetos, gestores e demais agentes envolvidos em novos projetos de edifícios educacionais, possam proporcionar ambientes para 0 desempenho compatível com as condicionantes emergentes na educação do século XXI.
\end{abstract}

Palavras-chave: Arquitetura Escolar, Inteligências Múltiplas, Metodologias Ativas.

\begin{abstract}
This article presents the discussion about the school in the 21 st century, given the impact of information and communication technologies. The research is based on the development of a doctoral thesis in architecture and urbanism, qualified at the end of the year 2018. The objective is to identify the new configurations of the school building, which will support the development of Multiple Intelligences (MI) through methodologies. In the methodology of research the research of digital and physical references was adopted, besides the study of cases to characterize the MI and the active methodologies. Thus, the different needs in the use of the school environment were identified and the projects of school buildings were evaluated, showing the new educational space scenario, publications of specialized magazines and visits in innovative schools. We conclude that a new configuration of the school environment, with a greater variety of learning environments, enhances the development of MI. Such environments should favor the most collaborative pedagogical practices in small learning communities, based on active methodologies. For this, the school building should be considered as a whole, from its access, to the environments for more specialized activities. Circulation spaces and eating places should also be incorporated as places of learning. New design guidelines for school buildings are
\end{abstract}

SQUAIELLA, R.; RIGHI, R. Edifício escolar voltado às inteligências múltiplas e às metodologias ativas. In: SIMPÓSIO BRASILEIRO DE QUALIDADE DO PROJETO NO AMBIENTE CONSTRUÍDO, 6., 2019, Uberlândia. Anais... Uberlândia: PPGAU/FAUeD/UFU, 2019. p. 1206-1214. DOI https://doi.org/10.14393/sbqp19110. 
needed for architects, managers and other agents involved in new educational building projects to provide environments for performance consistent with the emerging constraints on 21st century education.

Keywords: School Architecture, Multiple Intelligences, Active Methodologies.

\section{INTRODUÇÃO}

A nova dinâmica na educação, com maior diversidade e complexidade nas práticas pedagógicas, não pode mais ser reduzida ao ambiente escolar configurado para uma formação em massa, com salas de aula dispostas ao longo de um corredor, com poucos espaços especializados para atividades diferenciadas (PEREIRA et al., 2018). Infelizmente, verifica-se que a transformação deste modelo escolar obsoleto ocorre muito lentamente, apesar da emergência da mudança (SQUAIELLA; RIGHI, 2019). Entretanto, há a ascensão de propostas pedagógicas pontuais e relevantes, como as metodologias ativas, que visam um aprendizado cada vez mais personalizado e participativo do aluno, com grande integração do uso dos recursos tecnológicos. A intenção é o estudo e divulgação das experiências, visando transformação na configuração do ambiente educativo, atendendo às atuais e futuras demandas dos usuários do edifício escolar.

Geralmente a transformação do espaço de aprendizagem relaciona-se à escolha do mobiliário e ao uso de paredes móveis. Porém, o redesenho das salas de aula do século XXI deve ser mais profundo do que melhorar flexibilidade física, sendo o maior desafio dos prédios escolares responderem o ritmo das grandes transformações tecnológicas e sociais. Compreende-se que um espaço físico em si não é único responsável pela mudança da prática pedagógica, mas certos atributos desse espaço podem dar o apoio e estimular novas e diferentes atividades didáticas (LANGE, 2016), por meio de processos criativos e inovadores (KOHLERT; COOPER, 2017), que potencializam o desenvolvimento das inteligências múltiplas de cada um. Nesse sentido, Kowaltowski (2011) aponta que há uma relação fundamental entre aprendizado e arquitetura da escola, pois a qualidade do desempenho escolar é influenciada pelo edifício e suas instalações.

A teoria das inteligências múltiplas, de Howard Gardner (1994), é o principal enfoque da pesquisa, considerando a existência de diferentes inteligências que manifestam variadas habilidades de cada indivíduo e possibilitam que cada um aprenda de uma maneira que cumpra suas capacidades particulares. O uso das metodologias ativas resulta numa estratégia interessante para o desenvolvimento do aluno, partindo-se do pressuposto de que não existe uma única forma de aprender e, por consequência, não existe uma única forma de ensinar (MORAN, 2018).

Por meio de dois estudos de casos, são apresentadas as experiências de instituições de ensino que consideram a integração com as inovações tecnológicas para um aprendizado global e em ambientes preparados para as metodologias ativas. A necessidade de transformação na configuração do edifício escolar visa adequação das atividades e das propostas pedagógicas mais contextualizadas com a era das tecnologias digitais (SQUAIELLA; RIGHI, 2018). Deve-se salientar que esta pesquisa não se propõe à elaboração de um manual de arquitetura escolar, pois se acredita que o desempenho de um edifício escolar deve estar adequado a um conjunto complexo de variáveis 
que englobam as diretrizes pedagógicas, além das particularidades de cada cenário social e regional. Entretanto, a infraestrutura física e digital do espaço escolar é importante recurso para se proporcionar um aprendizado inovador.

\section{FUNDAMENTAÇÃO TEÓRICA}

\subsection{Teoria das Inteligências Múltiplas de Howard Gardner}

Em 1983, o neurologista e psicólogo do desenvolvimento Howard Gardner publicou sua teoria sobre Inteligências Múltiplas (IM), apoiado na psicologia e fisiologia do cérebro. Contrário aos testes de quociente de inteligência (QI), que se baseiam em questões linguística e lógico-matemática, Gardner sugeriu que a inteligência estivesse relacionada com a capacidade de resolver problemas e de criar produtos em um contexto rico e naturalista. Originalmente Gardner listou sete inteligências: linguística, lógico-matemática, espacial, musical, corporal-cinestésica, interpessoal e intrapessoal (GARDNER, 1994). Posteriormente, ele expandiu a lista acrescentando a inteligência naturalista e a possibilidade da inteligência existencial (GARDNER et al., 2010). Cada uma dessas inteligências manifesta variada habilidade em cada indivíduo e permite que cada um aprenda de uma maneira que cumpra suas intenções particulares.

Todas as pessoas possuem todas as inteligências, em diferentes graus de desenvolvimento, e não há dois seres humanos que possuam o mesmo perfil em suas qualidades e suas limitações em termos de inteligência, pois cada um passa por diferentes experiências e todos são motivados a se diferenciar um do outro, na busca de sua individualidade (GARDNER et al., 2010). A riqueza da teoria das IM está em enfatizar grande diversidade das pessoas mostrarem seus dons dentro de cada inteligência, bem como entre elas (ARMSTRONG, 2009). Destaca-se que a evolução dos dispositivos móveis torna a personalização da aprendizagem mais fácil e contribui sobremaneira para esse processo. Além disso, há várias formas de se ensinar qualquer ideia, disciplina ou conceito importante, que por meio de argumentos deve ativar diferentes inteligências ou combinações de inteligências (GARDNER et al., 2010).

\subsection{As metodologias ativas e o espaço de aprendizagem}

Uma escola considere a teoria das IM possibilita aos alunos desenvolver suas inteligências e competências com harmonia. De acordo com Smole (1999), uma educação baseada nas IM proporciona aos alunos: estímulo para 0 entendimento de algumas disciplinas básicas (como línguas, matemática, ciências, história, geografia e artes) utilizadas para a realização de tarefas dentro e fora da escola; incentivo para o desenvolvimento singular de inteligências em cada aluno; apoio da comunidade para a realização de atividades extracurriculares; ambiente onde os alunos se sintam livres para explorar novos estímulos e situações desconhecidas; engajamento dos alunos para a realização de projetos coletivos e individuais; e, ensinar aos alunos o registro do seu trabalho e processo de aprendizagem.

As formas tradicionais de aprendizagem, baseadas na oralidade do professor e na passividade do aluno, não atendem as expectativas da sociedade atual e do futuro. Para mudar esse quadro, é necessário que as metodologias 
pedagógicas contribuam para que os alunos envolvam-se em atividades mais complexas, exigindo a tomada de decisões e a avaliação dos resultados. Com as metodologias ativas busca-se desenvolver o protagonismo do aluno na construção do próprio conhecimento. Valente et al. (2017) destacam que, por meio de práticas ativas, os alunos são estimulados a: criar e investigar, ter pensamento crítico, saber se comunicar, desenvolver estratégias cognitivas, fornecer e receber o retorno do seu aprendizado, aprender e trabalhar em equipe e, explorar atitudes e valores pessoais e sociais. Assim, o aluno desenvolve as competências que mobilizam os conhecimentos, os saberes, as atitudes e os valores necessários para resolver os problemas reais.

Nessa era digital e conectada, as metodologias ativas se expressam por meio de modelos de ensino híbridos e flexíveis, que trazem importantes contribuições para a educação atual (MORAN, 2018). De acordo com Mccoog (2010), o primeiro passo para incorporar a teoria das IM em um currículo baseado em tecnologia, é avaliar as inteligências fortes e fracas de cada aluno. Entretanto, Armstrong (2009) ressalta que identificar o perfil das inteligências múltiplas de cada pessoa é uma tarefa difícil, pois nenhum teste é capaz de determinar com precisão quais são as inteligências de uma pessoa.

As metodologias ativas possibilitam o desenvolvimento das inteligências múltiplas ao aumentar a capacidade de se realizar diferentes tarefas, que se adaptam às diversas situações e, superam os modelos mentais rígidos e pouco eficientes. São exemplos de aprendizagem ativa: sala de aula invertida, design thinking, atividades de criação (maker), jogos e programação, aprendizagem baseada em investigação, em problemas e em projetos. De acordo com Smole (1999), para adotar um projeto que considere a teoria das IM, é importante analisar a forma de organização das atividades didáticas, avaliando aquelas mais adequada para cada fim.

\subsection{A transformação da sala de aula}

Analisando o impacto da teoria das IM na sala de aula, Armstrong (2009) avalia questões como o desenvolvimento de currículo, estratégias de ensino e aprendizagem e, o ambiente escolar. Sobre esse último, o autor sugere a sua reestruturação para acomodar a necessidade de diferentes tipos de atividades envolvidas na aprendizagem. Cada inteligência envolve um contexto que promove ou interfere na aprendizagem, sendo importante observar os elementos a serem incorporadas para o progresso do aluno. Se a qualidade do ambiente disponível para os alunos não contribui para 0 aprendizado significativo, o processo tende a ser prejudicado, mesmo que os alunos entrem na sala de aula com disposição, capacidade e entusiasmo para aprender. Por outro lado, um ambiente adequado tende a estimular o progresso na aprendizagem, mesmo para aqueles que possuem dificuldades acadêmicas, emocionais ou cognitivas significativas.

Uma possibilidade para se trabalhar com a teoria das IM nos espaços de aprendizagem é apontada por Armstrong como os centros de atividades. Neles, a organização didática ocorre agrupando as atividades dedicadas às inteligências específicas. Esses centros podem corresponder às salas de aulas, aos laboratórios e aos demais ambientes de aprendizagem (ARMSTRONG, 2009). Nesse sentido, identificam-se as metodologias ativas como estratégia pedagógica condizente com a variedade de atividades que apoiam 0 desenvolvimento das $I M$, e possibilitam a inter-relação entre atividades 
distintas. A multiplicação de recursos resultantes da combinação entre todas as IM suscitam novas e diferentes configurações espaciais para a aprendizagem. Considerando-se a grande convergência entre a teoria da IM e as metodologias ativas, são evidenciadas algumas questões relevantes para os futuros projetos de construção ou reforma de edifícios escolares.

\subsection{O edifício escolar para o aprendizado centrado no aluno}

Apoiado em quatro princípios de projeto, Nair (2017) defende que os edifícios escolares devem ser: receptivos; versáteis; apoiar várias atividades de aprendizado; e, enviar mensagens positivas sobre atividade e comportamento. Esses quatro princípios devem dar o apoio para as seguintes estratégias educacionais: 1. Aprendizagem centrada no aluno; 2. Colaboração de professores; 3. Clima escolar positivo; 4. Integração tecnológica; 5. Flexibilidade de horário; e, 6. Conexão ao ambiente, a comunidade e à rede global. Para alcançar tais estratégias educacionais, um edifício de aprendizagem deve proporcionar agilidade para as mudanças necessárias e inteligência na utilização dos recursos, respondendo às condições ambientais e aos estímulos do usuário.

Todo o edifício escolar deve estar comprometido com os princípios de projeto, onde as escolas têm ambientes, níveis de recursos e necessidades dos alunos muito diferentes. Envolve desde a importância das entradas, acolhedoras e receptivas, até os espaços de circulação, as salas de aula e as áreas comuns, suporte para o progresso incremental do aluno, a partir de várias atividades. As salas de aulas e os corredores de circulação devem ser redesenhados para criar comunidades de aprendizado, com a otimização dos espaços e minimizando o desperdício de áreas subutilizadas. Diferentes configurações espaciais possibilitam a colaboração entre professores que podem juntar turmas distintas, inclusive de diferentes idades. Além disso, as áreas específicas como os laboratórios, os estúdios especializados e os espaços para a criação, devem ser disponibilizados de maneira mais livre para todos os alunos. O papel da biblioteca se altera e se torna um lugar efetivo para reunir pessoas e ideias no mundo tecnológico atual. A importante conexão com a natureza pode ocorrer em terraços, hortas e ambientes ao ar livre ou, estratégias que tragam aspectos naturais, como plantas e animais, para o ambiente interno. Por fim, as grandes lanchonetes e refeitórios dão lugar aos pequenos cafés, mais centrados nas comunidades de aprendizagem, como local de estudos além das refeições. Para a maior eficiência da personalização, cada grupo de aprendizagem não deve ultrapassar 150 alunos com até 8 professores (NAIR, 2017).

\subsection{Estudo de Casos}

Apesar dos estudos apresentados não serem identificados como escolas para as inteligências múltiplas, oferecem uma proposta pedagógica e um espaço arquitetônico que condiz com o ensino personalizado, mais adequado às propostas da educação para o século XXI. A Avenues - The World School e a Escola Projeto Âncora adotam as metodologias ativas e os recursos das TIC para a participação ativa do aluno.

A Escola Projeto Âncora, localizada em Cotia/SP atende aproximadamente 180 alunos, desde a educação infantil (a partir dos 5 anos) até o ensino médio. A configuração do espaço escolar surgiu de programa assistencial, que não 
tinha como proposta inicial ser uma escola. Em visita realizada em 2017, verificou-se que a infraestrutura é composta por: salas de aprendizagem, salas de atividades corporais e de música, circo, quadra de esportes, biblioteca, refeitório, pista de skate, jardins com árvores frutíferas, canteiros de ervas e horta.

O foco está no processo de construção da autonomia, por meio de atividades colaborativas, que independem do espaço físico. Com o desenvolvimento do aluno, ele conquista maior liberdade para a realização das atividades onde for mais conveniente, podendo reduzir a sua presença física na escola, pois a plataforma virtual permite o acompanhamento das atividades realizadas à distância. Além disso, as parcerias estabelecidas na comunidade contribuem para o desenvolvimento de atividades diferenciadas, conforme a disponibilidade $e$ os interesses individuais, potencializando-se assim, o desenvolvimento das IM de cada um.

Cada sala de aprendizagem possui uma conformação e tamanho diferenciado, onde os mobiliários escolares geralmente são arranjados para os estudos individuais, em duplas ou em pequenos grupos. Dispostas em diferentes áreas da escola, não se encontram salas de aula enfileiradas ao longo de um corredor. Há grande integração entre os ambientes interno e externo, que propicia o contato com as áreas verdes, benéfico para restauração cognitiva.

A Avenues - The World School é integra rede de escolas norte americana que possui uma unidade na cidade de São Paulo/SP. Possui capacidade para atender até 2.100 alunos, desde a educação infantil (3 anos) até o ensino médio. A escola divide-se em quatro programas, organizados em espaços distintos, distribuindo os alunos conforme a faixa etária. De acordo, com a análise do projeto arquitetônico do escritório Aflalo e Gasperini, verifica-se que as atividades estão distribuídas em dois blocos principais, sendo um bloco menor, destinado às atividades culturais e esportivas, e o outro maior, onde se concentram as atividades didáticas. Visando facilitar a participação e a interação entre os alunos e os professores, há a combinação da formação presencial com o poder das tecnologias emergentes. Isto possibilita 0 aprendizado em ambiente internacionalmente colaborativo, considerando-se a relação entre todas as unidades da instituição. Apesar das turmas estarem divididas em grau etário, como ocorre na educação tradicional, observa-se que as salas de aula estão configuradas para o desenvolvimento de diferentes atividades como o trabalho em grupo, os momentos de descanso e a exploração de atividades individuais. Para as atividades de STEAM (ciência, tecnologia, engenharia, arte e matemática) há laboratórios específicos, que possuem amplas aberturas com função de inspirar e instigar a curiosidade a respeito das atividades que ocorrem internamente, entre os grupos. As atividades complementares ocorrem em grandes espaços específicos, tais como: teatro, ginásio, pátio coberto, quadras esportivas e áreas externas descobertas.

A grande variedade de espaços e escalas dos mobiliários e dos ambientes tem a função de adaptar as atividades às diversas necessidades educacionais de cada faixa etária. As áreas comuns e os espaços de circulação visam incentivar os trabalhos colaborativos, as leituras individuais ou para promover a convivência entre os alunos. No pavimento térreo há espaços sociais, como o 
café, nos quais os pais e professores podem circular livremente, contribuindo para a integração da família no processo de aprendizagem.

\section{METODOLOGIA}

Foram adotadas como metodologia de pesquisa as seguintes etapas:

1. Pesquisa de referências digitais e físicas que evidenciaram:

- Importância da teoria das inteligências múltiplas na identificação e desenvolvimento das potencialidades individuais de cada estudante.

- Potencialidade das metodologias ativas como prática pedagógica inovadora, dando o apoio necessário para o desenvolvimento de um aprendizado personalizado.

- Necessidade de redesenho do espaço escolar para adequação das propostas pedagógicas mais atuais, com o aprendizado centrado no aluno.

2. Estudos de casos de escolas inovadoras, a partir de publicações em revistas especializadas e visitas em escolas inovadoras, que apresentam diferentes configurações do espaço de aprendizagem.

3. Análise das experiências apresentadas nos estudos de casos para 0 estabelecimento de diretrizes projetuais para a concepção de futuros projetos arquitetônicos em edifícios escolares.

\section{RESULTADOS E DISCUSSÃO}

Os ambientes de aprendizagem suscitam uma nova configuração que apoie as atividades mais colaborativas e significativas para o aluno. Isso significa romper com as salas de aula configuradas com carteiras de alunos enfileirados diante de um professor. Destacando-se o potencial das IM no desenvolvimento de cada um, é preciso considerar o aluno como protagonista no processo de aprendizagem. Nesse sentido, as metodologias ativas contribuem para a personalização da aprendizagem, com uma variedade de atividades que demandam maior envolvimento.

Apesar dos estudos de casos não apresentarem escolas que se identifiquem como seguidoras da teoria das IM considera-se que o real valor do aprendizado centrado no aluno está na sua capacidade de ser personalizado (NAIR, 2017), o que vai de encontro com a teoria das IM ao considerar as particularidades de cada aluno.

Para o edifício escolar dar o suporte à aprendizagem do aluno de forma inovadora, ele deve ser adaptado às necessidades dos alunos e dos professores à medida que as necessidades evolvem. Nesse sentido, os ambientes de aprendizagem devem ser variados e confortáveis, a fim de ampliar a gama de métodos de ensino e aprendizagem disponíveis (NAIR, 2017).

Compreende-se que as inteligências múltiplas se desenvolvem em ambientes variados, o que significa favorecer as comunidades de aprendizagem, onde as práticas pedagógicas possam ocorrem de maneira mais colaborativa. Tanto os professores, quanto os alunos, se apoiam em seus pares para alcançar melhores resultados na aprendizagem. Por meio das metodologias 
ativas os alunos são instigados a participar ativamente do seu processo de aprendizagem, o que potencializa o desenvolvimento das suas inteligências.

O edifício escolar deve ser considerado como um todo, desde o seu acesso, até os ambientes para atividades mais especializadas. Centros de atividades específicas podem fornecer os subsídios para o desenvolvimento de cada uma das IM. Espaços de circulação e local para refeições também devem ser incorporados como locais de aprendizagem, oferecendo maior flexibilidade e possibilidade para que o aprendizado ocorra maneira formal ou informal.

\section{CONSIDERAÇÕES FINAIS}

Para a adequação da escola à sociedade do século XXI, são necessárias profundas transformações das quais se destacam os métodos pedagógicos e o redesenho do espaço escolar. O projeto de edifícios escolares é um campo que abrange teorias educacionais em constante mudança, sendo necessária a interpretação e antecipação das necessidades específicas de novos métodos de aprendizagem.

A partir da teoria de Gardner considera-se que há uma multiplicidade de inteligências na construção do conhecimento ao longo da vida de cada estudante, que se desenvolve a partir de uma combinação de fatores biológicos, culturais e sociais. Dessa maneira, considera-se a importância do edifício escolar para dar o suporte adequado ao desenvolvimento das inteligências múltiplas de cada aluno.

Destaca-se que, atualmente, as propostas pedagógicas devem considerar a maior integração com as TIC, pois a evolução dos dispositivos móveis torna mais viável a personalização da aprendizagem. Nesse sentido, identifica-se que as metodologias ativas possibilitam o desenvolvimento das inteligências múltiplas ao aumentar a capacidade de se realizar diferentes tarefas, que se adaptam as mais diversas situações e, superam os modelos mentais rígidos e pouco eficientes.

Novas diretrizes projetuais de edifícios escolares são necessárias para que arquitetos, gestores e demais agentes envolvidos em novos projetos de edifícios educacionais, possam proporcionar ambientes compatíveis com as condicionantes emergentes na educação do século XXI. Deve-se permitir que os alunos tenham tempo e espaço para escolher o que querem fazer, considerando-se que suas escolhas podem potencializar as suas forças individuais.

\section{REFERÊNCIAS}

ARMSTRONG, T. Multiple Intelligences in the Classroom. 3 ed. Virginia/EUA: ASCD, 2009.

GARDNER, H. Estruturas da mente: a Teoria das Múltiplas Inteligências. Tradução Sandra Costa. Porto Alegre: Artes Médicas Sul, 1994.

KOHLERT, C. E.; COOPER, S. M. Space for Creative Thinking: Design Principles for Work and Learning Environments. Callwey, 2017.

KOWALTOWSKI, D. K. Arquitetura escolar e o projeto do ambiente de ensino. São Paulo: Oficina de textos, 2011. 
LANGE, C. Architecture's Pivotal Role in the Future of K-12 Learning. EdSurge, 2016.

MCCOOG, I. J. Integrated Instruction: Multiple Intelligences and Technology. The Clearing House: A Journal of Educational Strategies, 2010.

MORAN, J. M. Metodologias ativas para uma aprendizagem mais profunda. In: BACICH, L.; MORAN, J. (org.). Metodologias Ativas para uma Educação Inovadora: Uma Abordagem Teórico-Prática. Penso Editora, 2018.

NAIR, P. Blueprint for tomorrow: Redesigning schools for student-centered learning. 3 ed. Cambridge, MA: Harvard Education Press, 2017.

PEREIRA, P. R. P.; KOWALTOWSKI, D. C. C. K.; DELIBERADOR, M. S. Analysis support for the design process of school buildings. Ambiente Construído, v. 18, n. 3, p. 375-390, 2018.

SMOLE, K. C. S. Múltiplas Inteligências na Prática Escolar. Brasília: Ministério da Educação, Secretaria de Educação a Distância, 1999.

SQUAIELLA, R. B. F.; RIGHI, R. Repensando o espaço construído da edificação escolar com as práticas pedagógicas inovadoras. In: Encontro da Associação Nacional de Pesquisa e Pós-Graduação em Arquitetura e Urbanismo, 4., 2018. Anais V ENANPARQ, Salvador/BA: FAUFBA, 2018.

SQUAIELLA, R. B. F.; RIGHI, R. Inteligências múltiplas e as metodologias pedagógicas ativas na escola do século XXI. IN: MARTINS, B. C. (org.). O essencial da Arquitetura e Urbanismo 2. Ponta Grossa (SP): Atena Editora, 2019.

VALENTE, J. A.; DE ALMEIDA, M. E. B.; GERALDINI, A. F. S. Metodologias ativas: das concepções às práticas em distintos níveis de ensino. Revista Diálogo Educacional, v. 17, n. 52, 2017. p. 455-478. 